\title{
Acquired Congenital Malalignment of the Great Toenails
}

\author{
Ashley Decker Richard K. Scher Andrew Avarbock \\ Department of Dermatology, Weill Cornell/New York Presbyterian, New York, N.Y., USA
}

\section{Key Words}

Nail disease · Nail disorder · Nail surgery

\begin{abstract}
Congenital malalignment is the lateral deviation of the nail plate along the longitudinal axis due to the lateral rotation of the nail matrix. The nail plate grows out in ridges caused by repeated microtrauma to the nail. Common complications include onychomycosis, Pseudomonas infection and acute or chronic paronychia. Treatment options range from conservative management to surgical options including realignment and nail matrixectomy. Congenital malalignment usually presents in infancy or childhood, but we present two cases of acquired malalignment occurring in the teenage years.

(c) 2015 S. Karger AG, Basel
\end{abstract}

\section{Introduction}

Congenital malalignment of the great toenail was first described by Samman [1] in 1978 and then further depicted by Baran et al. [2] in 1979 as the lateral deviation of the nail plate along the longitudinal axis due to lateral deviation of the nail matrix. This uncommon entity usually presents in infancy or childhood; late onset is uncommon. Treatment options range from conservative to surgical management depending on the severity of the case.

\section{Case 1}

A 21-year-old female with reflex neurovascular dystrophy presented to our clinic with a 6-year history of nail dystrophy involving both halluces (fig. 1). At the age of 10 years, the patient began experiencing severe joint pain. After several years and extensive workups, she was diagnosed with reflex neurovascular dystrophy and underwent intensive physical therapy with marked improvement in her symptoms. At the age of 16 years, her great toenails became tender and erythematous with a purulent discharge. Up until that point, the patient's toenails were clinically normal and there was no history of trauma. The patient was treated for an infection, as bacterial culture revealed Enterobacter, which on initial dermatologic evaluation was diagnosed as pernio. Since that time, the patient has been seen by several other health care units including dermatology and podiatry. Additional treatments included total nail avulsion of the left hallux and topical antifungals, without any improvement in her symptoms. There is a family history of psoriasis in her maternal aunt and cousin.

Physical examination showed yellow discoloration, full-length thickening of both halluces, Beau's lines extending along the length of the nail, a disappearing nail bed [3] and lateral deviation of the

\section{KARGER 125}

(c) 2015 S. Karger AG, Basel

0000-0000/15/0013-0147\$39.50/0 


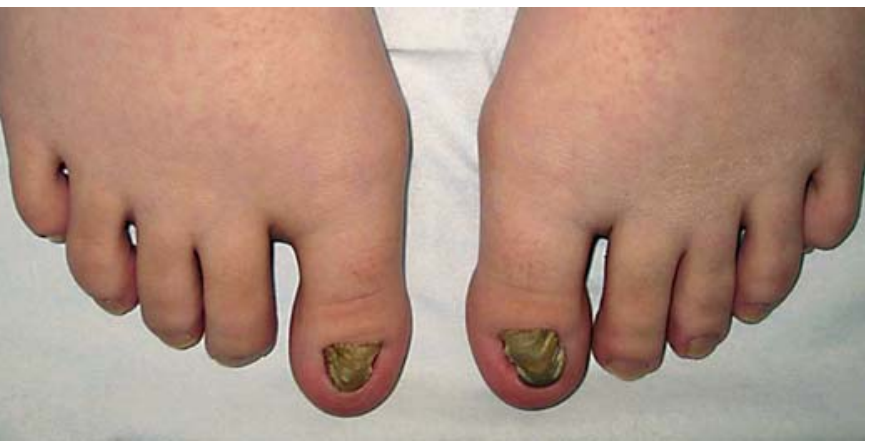

Fig. 1. Case 1 showing yellow discoloration, full-length thickening of both halluces, Beau's lines extending along the length of the nail, a disappearing nail bed [2] and lateral deviation of the nail plate.

nail plate. Imaging was not performed. A diagnosis of acquired malalignment of the great toenails was made. Additional treatments were declined as quality of life was unaffected at this time and cosmetic appearance was acceptable.

\section{Case 2}

A 20 -year-old female with no past medical history presented with discoloration of the great toenails bilaterally starting at the age of 16 years (fig. 2). Prior treatments included a course of terbinafine (as per the patient, nail clippings were 'positive for fungus', we were unable to obtain previous medical records) and avulsion of both toenail plates; neither treatment improved the patient's symptoms. At the time of presentation, the patient was using econazole nitrate cream daily as well as soaking her feet in warm water with baking soda. She denied any history of trauma.

Physical examination showed lateral deviation of both great toenail plates with a blue-green discoloration. Beau's lines extending along the width of the nail were present. The palms, soles and other nails were normal. A nail clipping specimen from the right toe was sent for PAS stain and was positive for fungal hyphal elements and scattered Gram-negative rod-shaped bacteria. Imaging was not performed.

The condition was diagnosed as acquired malalignment of the great toenails along with onychomycosis and secondary retronychia. The authors hypothesize that the underlying malalignment led to repeated trauma causing retronychia. Topical treatment with clotrimazole and urea cream was prescribed. At the time of the visit, the patient was considering surgical correction.

\section{Discussion}

Congenital malalignment of the great toenails is characterized by lateral deviation of the nail plate along the longitudinal axis due to lateral rotation of the nail matrix [1]. Medial deviation has also been reported [4]. Ma-

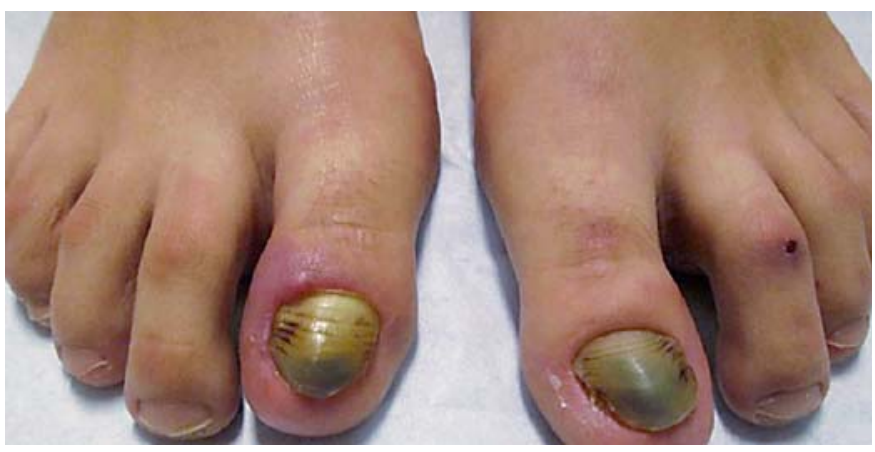

Fig. 2. Case 2 showing lateral deviation of both great toenail plates with a blue-green discoloration and Beau's lines extending along the width of the nail.

lalignment may be unilateral or bilateral and most often affects the halluces but also other toenails. The nail plate grows out in transverse ridges caused by repeated microtrauma to the nail matrix. Discoloration is a common finding, which can be exacerbated by infection or hemorrhage [5]. Common opportunistic infections of the nail plate include fungal and/or Pseudomonas infection. The malaligned toenail frequently becomes imbedded in the lateral nail fold leading to inflammation, erythema and pain. Acute or chronic paronychia is another frequent complication that exacerbates ingrowth.

The actual pathogenesis of congenital malalignment is unknown, but several hypotheses exist including lateral rotation of the nail matrix, an abnormality in the ligament that connects the matrix to the periosteum of the distal phalanx and environmental causes (i.e. intrauterine or vascular insult during fetal growth) [6]. A new hypothesis by Chaniotakis et al. [6] suggested desynchronization in growth between the nail and the distal phalanx of the hallux leading to larger nail plates, which must grow laterally to fit into the underlying bony space.

Acquired forms may be caused by mechanical or surgical trauma. A recent retrospective study by Kim et al. [7] has looked at the clinical features of 21 patients with acquired great toenail dystrophy [7]. A preceding history of trauma was identified in 12 of 21 (57.1\%) cases with development of dystrophy [7]. The authors hypothesize that trauma was the inciting factor leading to the development of acquired malalignment in the cases presented above.

Congenital ectodermal dysplasia syndromes, such as pachyonychia congenita, present with malformation of the nails but are distinguished by involvement of other ectodermal structures in addition to the nail dystrophy. 
There is a wide range of treatment options depending on the severity of the condition and the complications. Conservative choices should be tried initially, especially as approximately $50 \%$ of the cases will spontaneously improve. Wearing proper-fitting, comfortable footwear should be recommended to decrease the risk of repeated trauma. Surgery is performed in severe cases and involves rotating the nail matrix to the proper orientation allowing the nail plate to grow in parallel to the distal phalanx [8]. A wedge-shaped excision follows the curvature of the distal toe. The excision is extended 3-4 mm down the lateral aspect of the dorsal toe. The nail unit is dissected away from the bone, and the nail matrix is realigned and sutured into place. Surgical correction is recommended until up to 2 years of age, although this cutoff is flexible [8].

Nail matrixectomy is another surgical option, which involves complete removal of the nail plate and matrix. Removal of the nail unit leads to complete resolution of symptoms but may not be cosmetically acceptable.
In the two cases presented, both patients developed symptoms consistent with acquired malalignment during adolescence. This is an uncommon entity as most cases develop during infancy. Despite the delayed onset, treatment options remain the same and range from conservative management to surgical correction. Spontaneous correction is less likely to occur in these patients and thus surgical correction is a viable option depending on the patient's preference.

\section{Statement of Ethics}

The patients' consent has been obtained.

\section{Disclosure Statement}

The authors have no conflicts of interest to disclose.

\section{References}

Acquired Malalignment
1 Samman PD: Great toenail dystrophy. Clin Exp Dermatol 1978;3:81-82.

2 Baran R, Bureau H, Sayag J: Congenital malalignment of the big toe nail. Clin Exp Dermal 1979;4:359-360.

3 Daniel CR 3rd, Tosti A, Iorizzo M, Piraccini BM: The disappearing nail bed: a possible outcome of onycholysis. Cutis 2005;76:325327.

4 Baran R, Bureau H: Congenital alignment of the big toenail: a new subtype. Arch Dermatol 1987; 123:437.

5 Perlis CS, Telang GH: Congenital malalignment of the great toenails mimicking onychomycosis. J Pediatr 2005; 146:575.
6 Chaniotakis I, Bonitsis N, Stergiopoulou C, Kiorpeliduo D, Bassukas I: Dizygotic twins with congenital malalignment of great toenails: reappraisal of the pathogenesis. J Am Acad Dermatol 2007;57:711-715.

7 Kim M, Kang JH, Cho BK, Song CH, Ock SM, Park HJ: Great toenail dystrophy: a singlecenter experience and review of the literature. Korean J Fam Med 2015;36:113-120.

8 Baran R, Haneke E: Etiology and treatment of nail malalignment. Dermatol Surg 1998;24: 719-721. 